\title{
Comparación antropométrica, fuerza explosiva y agilidad en jugadoras jóvenes de baloncesto de Bogotá- Colombia \\ Anthropometric comparison, explosive strength, and agility in young basketball players from Bogotá- Colombia \\ Yennys González de los Reyes, Angela Yazmín Gálvez Pardo, Dario Mendoza Romero \\ Universidad Santo Tomás (Colombia)
}

\begin{abstract}
Resumen. Objetivo: Comparar las características antropométricas, la fuerza explosiva y la agilidad en las categorías infantil, prejuvenil y juvenil entre dos asociaciones competitivas de baloncesto femenino de la ciudad de Bogotá. Métodos: El estudio tuvo un enfoque cuantitativo, con diseño no experimental de tipo transversal correlacional, ya que se compararon los resultados antropométricos, de fuerza explosiva y agilidad en jugadoras del nivel liga departamental (GL1) y el nivel de colegios internacionales de Bogotá, Uncoli (GC2). En cada uno de estos niveles se estudiaron tres categorías: infantil (sub-12), prejuvenil (sub-14) y juvenil (sub-16). Resultados: El grupo GL1 entrena 6 horas a la semana y el GC2 lo hace por 4 horas. Ambos tienen un partido el fin de semana. El GC2 presentó mejores resultados en las pruebas de T-Test y salto en contra movimiento con respecto al GL1 ( $\mathrm{p}<0,05)$. Mediante un análisis de regresión lineal se encontraron relaciones en la categoría juvenil entre el resultado del T-Test por izquierda y el porcentaje de grasa $\left(r^{2}=0.47 ; F_{1,31}=27.39\right.$; $\mathrm{p}=0.001$ ). Conclusiones: El grupo de colegios privados presentó mejores valores de fuerza explosiva y agilidad con respecto al grupo de liga departamental, lo que permite sugerir que un mayor volumen de entrenamiento no necesariamente mejora este indicador.

Palabras clave: Baloncesto, Fuerza, agilidad, antropometría y jugadoras jóvenes.
\end{abstract}

Abstract. Objective: To compare anthropometrics, explosive strength, and agility characteristics in infantile, pre-juvenile, and juvenile categories between two competitive associations of women's basketball in the city of Bogotá. Methods: cross sectional study, since anthropometric and physical variables were compared at two competitive levels of basketball in the city of Bogotá, departmental league level (GL1), and level of international private schools (GC2). At each of these levels, three categories were studied: infant (sub-12), prejuvenile (sub-14), and juvenile (sub-16). Results: The GL1 group trains 6 hours a week and the GC2 trains for 4 hours. They both have a game over the weekend. The GC2 presented better results in the T-Test and countermovement jump tests with respect to GL1 (p $<0.05$ ). By means of a linear regression analysis, relationships were found in the juvenile category between the result of the T-Test on the left and the percentage of fat $\left(\mathrm{r}^{2}=0.47, \mathrm{~F} 1.31=27.39, \mathrm{p}=0.001\right)$. Conclusions: The group of private schools presented better values of power, strength, and agility than the departmental league group, which suggests that a higher volume of training does not necessarily improve this indicator.

Keywords: Basketball, strength, agility, anthropometric and young players.

\section{Introducción}

En el área de la educación física los deportes de cooperación y oposición como el baloncesto, son de los más practicados y preferidos por los niños en el contexto escolar tanto en la básica primaria como secundaria (González Espinosa et al., 2017; Otero et al., 2014).

Por otro lado, es uno de los deportes más practicados en el mundo, especialmente por mujeres y se caracteriza por movimientos que requieren fuerza explosiva, agilidad con y sin balón y velocidad de movimientos cíclicos y acíclicos (Garcia-Gil et al., 2018; Narazaki et al., 2009). El jugador de baloncesto presenta características antropométricas que lo diferencia del resto de los deportistas, son más altos, más pesados y con extremidades más largas, siendo estos indicadores junto con el porcentaje de grasa, diámetros corporales, entre otros, determinantes para el rendimiento en esta disciplina (Vaquera et al., 2015).

Las características antropométricas también son determinantes para definir los procesos de selección y orientación deportiva. Dependiendo de los resultados de este tipo de indicadores, los deportistas pueden tener mayor o menor rendimiento en pruebas que demanden fuerza, resistencia o agilidad (Masanovic, 2018). Por ejemplo, se ha evidenciado

Fecha recepción: 04-04-19. Fecha de aceptación: 13-02-20

Dario Mendoza Romero

dariomendoza@usantotomas.edu.co que dependiendo de la posición de juego habitual en la cancha de juego, los jugadores de baloncesto presentan algunas diferencias en su composición corporal, los aleros y centros son por lo general más altos, pesados y con un mayor porcentaje de grasa con respecto a los escoltas (Ostojic et al., 2006). Sin embargo, en categorías juveniles éstás diferencias pueden ser sutiles (Peña, Moreno-Doutres, Coma, \& Cook, 2018), así como también las relaciones entre variables físicas y antropométricas no son necesariamente fuertes (Pizzigalli, Micheletti, La Torre, Rainoldi, \& Benis, 2017).

Con relación a las capacidades físicas, la agilidad, según, la Asociación Nacional de Fuerza y Condicionamiento, más conocida por sus siglas e iniciales en inglés, NSCA es la habilidad de frenar explosivamente, cambiar de dirección, y acelerar nuevamente (Baechle \& Earle, 2008). La agilidad no tiene una definición global, pero es identificada con frecuencia como la capacidad para cambiar de dirección, arrancar y detenerse rápidamente (Little \& Williams, 2005). En cuanto a la fuerza explosiva es la capacidad que tiene el músculo para desarrollar gradientes o desniveles de fuerza elevados en un espacio de tiempo breve (Bosco, 2000). Este mismo autor menciona que este tipo de fuerza depende de muchos factores como puede ser el tipo de movimiento, las condiciones anteriores del músculo (reposo o pre-estiramiento) y sus características morfológicas (tipo de fibras), el nivel de entrenamiento del individuo, sus características nerviosas (frecuencia de impulsos, sincronización y coordinación intermuscular) y la situación hormonal en ese momento. 
La fuerza explosiva en el baloncesto ha demostrado tener una relación con el rendimiento, especialmente lo que se refiere a la efectividad en el lanzamiento al aro, sin embargo, pruebas de agilidad como el T Test no han mostrado esta relación (Pojskic et al., 2018), a pesar de la existencia de asociación que pueda tener estas dos capacidades (Braz et al., 2017) y del grado de desarrollo cognitivo que debe tener el jugador para que se de esta relación (Young et al., 2015). Ahora bien, no se han llevado a cabo muchos estudios donde se relacione estas variables con distintas medidas antropométricas en jugadores en formación de distintos niveles competitivos, especialmente con mujeres jóvenes en contextos latinoamericanos.

Por estas razones, el presente estudio busca comparar las características antropométricas, de fuerza explosiva y agilidad en practicantes de Baloncesto, ambos competitivos, pero con distinto volumen de horas de entrenamiento a la semana en las categorías infantil, prejuvenil y juvenil de clubes pertenecientes a la ciudad de Bogotá.

\section{Materiales y métodos}

El presente estudio tuvo un enfoque cuantitativo, con diseño no experimental observacional analítico (Blanco \& Maya, 2008), ya que se compararon los resultados antropométricos, de fuerza explosiva y agilidad en jugadoras del nivel liga departamental (GL1) y el nivel de colegios internacionales de Bogotá, Uncoli, (GC2). En cada uno de estos niveles se seleccionan tres categorías, infantil (sub-12), prejuvenil (sub-14) y juvenil (sub-16). El GL1 entrenaba 6 horas a la semana, más una competencia el fin de semana, mientras que GC2 entrena 4 horas a la semana y un partido el fin de semana. Estos grupos se encontraban en el periodo preparatorio, etapa de preparación física general.

\section{Participantes}

El muestreo fue no probabilístico; se evaluaron ochenta jugadoras de baloncesto, distribuidas en tres categorías, 14 en la categoría infantil, edad promedio $11,64( \pm 0,49$ años), peso promedio $48,0 \mathrm{~kg}( \pm 9,71 \mathrm{~kg})$, talla promedio $1,53 \mathrm{~m}( \pm 0,09$ $\mathrm{m}) ; 30$ en la categoría prejuvenil, edad promedio 14,4 ( $\pm 0,49$ años), peso promedio $52,7 \mathrm{~kg}( \pm 7,93 \mathrm{~kg})$, talla promedio 1,59 $\mathrm{m}( \pm 0,07 \mathrm{~m})$ y 36 en la categoría juvenil, edad promedio 16,17 $( \pm 1,08$ años $)$, peso promedio $57,31 \mathrm{~kg}( \pm 9,00 \mathrm{~kg})$, talla promedio 1,60 m $( \pm 0,06 \mathrm{~m})$. Las jóvenes Basquetbolistas provenían de dos asociaciones competitivas en Bogotá, 46 de liga departamental y 34 del sector asociado de colegios internacionales de Bogotá, Uncoli. Las jugadoras de la liga departamental de Bogotá pertenecían a la escuela de formación deportiva de baloncesto del club Pumas y las del sector asociado de colegios internacionales son jugadoras de un campeonato pertenecientes a una organización privada llamada, Uncoli. Tanto las jugadoras, como los entrenadores y padres de familia fueron informados claramente sobre el objetivo, procedimiento y pruebas a realizar con las jóvenes, además de los posibles riesgos y beneficios del estudio. Posteriormente, otorgaron y firmaron los consentimientos informados acorde a la Declaración de Helsinki. El estudio fue aprobado por el comité de investigaciones de la Universidad Santo Tomás de Bogotá.

\section{Procedimiento}

Las pruebas de aptitud física y antropométrica se llevaron a cabo en las instalaciones deportivas de la unidad deportiva del salitre en Bogotá y en diferentes escuelas de formación deportiva de este deporte, en el período preparatorio, etapa de preparación física, de las diferentes categorías. Todos los grupos, antes de las pruebas de campo y en el laboratorio, realizaron un calentamiento estandarizado de 20 minutos. Todas las pruebas antropométricas, de campo y de laboratorio se realizaron en dos sesiones a la misma hora de la mañana y con los mismos parámetros.

Las pruebas antropométricas utilizadas fueron peso, estatura, cinco pliegues (tríceps, subescapular, supraespinoso, muslo y pantorrilla), diámetros (muñeca, fémur, húmero) y perímetros (codo flexionado del brazo, pantorrilla). Estos se realizaron de acuerdo con las normas ISAK; para la talla, se utilizó la varilla de medición Holtain $(60-209 \mathrm{~cm} ; 0,1 \mathrm{~cm})$ para las escalas de masa corporal Tanita BF-666 (0,1 kg 0-150 kg), los pliegues se midieron con calibrador Harpenden modelo modelo HSK-BI (0-80 mm; precisión de $0.20 \mathrm{~mm}$ ) diámetro por marca LYW tolos $(0.5$ a $110 \mathrm{~cm}$, precisión $1 \mathrm{~mm})$. La persona encargada de realizar estas medidas es certificada por el ISAK.

Luego de la evaluación antropométrica, se realizó un calentamiento de 20 minutos en una cancha de baloncesto, se aplicaron las pruebas de fuerza del tren superior con ayuda del lanzamiento de balón medicinal, LBM (Martinez, 2002), medido con un balón de $2 \mathrm{~kg}$ de peso. Posterior a esta prueba se valora la agilidad por medio del T-test (Pauole et al., 2000) que se mide con un sistema de fotocélula luz infrarroja modelo WL34 - R240, marca Sick (tiempo de retardo: tabla, ajustable de 0,5 a $10 \mathrm{~s}$ ). Por último, se realizaron las pruebas de fuerza explosiva en tren inferior con ayuda del test de salto en contra movimiento, CMJ, y el salto Abalakov, ABK, (Bosco \& Riu, 1994) con el sistema óptico de datos OptoGait que emplea el software OptoGait versión 1.12.1.0. de Microgate ${ }^{\circledR}$.

\section{Análisis estadísticos}

Los datos son presentados con medias y desviaciones estándar, sin embargo, se realizaron pruebas de normalidad (Kolmogorov Smirnov o Shapiro Wilk) y en los casos dónde la distribución es asimétrica, se emplean pruebas bivariadas comparativas sobre las medianas. Es decir, se aplica la prueba de T-Student para comparar los grupos GL1 y GC2 en cada categoría cuando los datos son paramétricos y la prueba U de Mann Whitney cuando no son paramétricos. Las relaciones entre variables son analizadas con el índice de correlación de Pearson (r) y un modelo de regresión con la variable de mayor ajuste fue aplicado. El nivel de significancia asignado es de $\mathrm{p}<0,05$ y los datos fueron procesados en el software IBMSPSS versión 25 (Licencia Universidad Santo Tomás).

\section{Resultados}

En primer lugar, se muestran los resultados descriptivos de las variables de edad, peso y talla para las tres categorías en los grupos GL1 y GC2 (tabla1). Se aplicaron pruebas de normalidad por categoría y nivel en estas variables, obteniendo una distribución asimétrica para la edad (Shapiro- 
Wilk; $\mathrm{p}<0,01)$. Por tanto, las estimaciones puntuales reportadas son obtenidas mediante la prueba de Mann Whitney y T-student para el peso y la talla.

En las tablas 2 y 3 se muestra la comparación por GL1 y GC2 de las variables antropométricas y pruebas físicas aplicadas a las 80 jugadoras de Baloncesto en las tres categorías.

En la categoría infantil se encontraron diferencias en el peso graso, peso muscular, porcentaje óseo e índice de masa corporal $(p<0,05)$, siendo mayores en GL1 a excepción del porcentaje óseo. En la categoría prejuvenil el porcentaje óseo es mayor en $\mathrm{GC} 2,18,23( \pm 1,85)$ frente a $16,25( \pm 2,05)(\mathrm{p}=0,01)$. El comportamiento de las variables en la categoría juvenil fue similar, siendo mayores los resultados de los pliegues de muslo y porcentaje graso en el grupo GL1 $(p<0,01)$, mientras que el porcentaje y peso óseo es mayor en GC2 ( $<<0,05)$.

En las pruebas físicas se observa un mejor rendimiento del CMJ en las tres categorías en GC2 $(p<0,01)$, mientras que en la categoría juvenil el salto Abalakov fue también mayor en $\mathrm{GC} 2(\mathrm{p}=0,034)$. El lanzamiento de balón medicinal el grupo GC2 se destacó frente al GL1 en la categoría prejuvenil (4,13 m y 3,69 m respectivamente, $\mathrm{p}=0,01)$. La categoría juvenil en la prueba $\mathrm{T}$ test por derecha e izquierda se encontró que el GC2 obtuvo menores tiempos que el GC1 $(\mathrm{p}<0,01)$.

En la categoría juvenil se realizan correlaciones entre las variables físicas y antropométricas, teniendo en cuenta que el número de sujetos evaluados es mayor en este grupo obteniéndose valores de correlación de Pearson moderados y que se detallan en la tabla 4.

Se realiza un modelo de regresión lineal por pasos, tomando como variable dependiente el $\mathrm{T}$ test por izquierda, en la categoría juvenil con los datos de ambos grupos, GL1 y GC2, obteniendo una relación significativa con el porcentaje graso $\left(\mathrm{r}^{2}=0,47 ; \mathrm{F}_{1,31}=27,39 ; \mathrm{p}=0,001\right)$ que se describe por medio de la ecuación presentada en la figura 1. Este modelo cumplió con los supuestos de distribución normal de los residuos (Kolmogorov-Smirnov $=0,075 ; \mathrm{p}=0,2$ ), homocedasticidad y el estadístico de Durbin-Watson no representa autocorrelación de los residuos. Se eliminaron las variables de pliegue de pantorrilla, pliegue de muslo y porcentaje muscular.

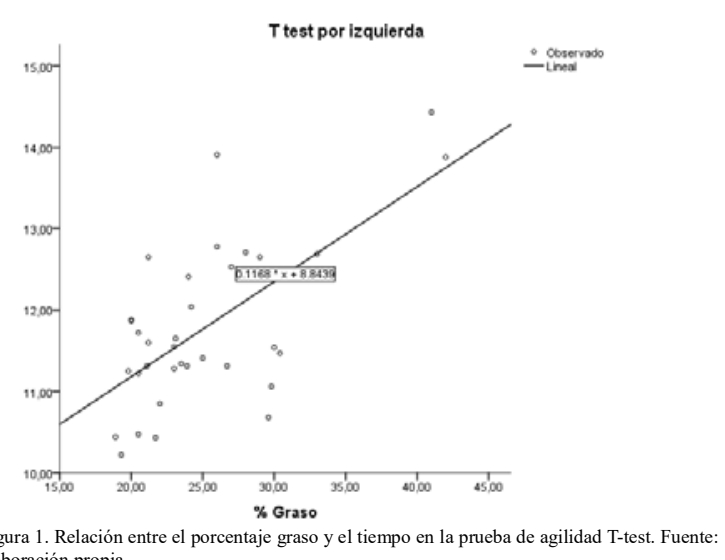

Figura 1. Relación
elaboración propia.

\section{Discusión}

El nivel de rendimiento en el baloncesto está influenciado no solamente por la capacidad de ejecución de los practican-
Tabla 1.

Características en edad, peso y talla por categoría y nivel. *Diferencias significativas entre los niveles de Liga y Escolar mediante la prueba de T-student (a) o la prueba U de Mann-Whitney (b).

\begin{tabular}{ccccccccc}
\hline \multirow{2}{*}{ Categoría } & Nivel & \multicolumn{4}{c}{ GL1 } & \multicolumn{4}{c}{ GC2 } & \\
\cline { 3 - 8 } & Variable & $\mathrm{n}$ & Media & $\begin{array}{c}\text { Desviación } \\
\text { estándar }\end{array}$ & $\mathrm{n}$ & Media & $\begin{array}{c}\text { Desviación } \\
\text { estándar }\end{array}$ & Valor $\mathrm{p}$ \\
\hline \multirow{3}{*}{ Infantil } & Edad (años) & & 11.67 & 0.50 & & 11.60 & 0.55 & $0.898 \mathrm{~b}$ \\
& Peso (kg) & \multirow{2}{*}{9} & 52.86 & 8.19 & 5 & 39.28 & 4.93 & $0.006 \mathrm{a}^{*}$ \\
& Talla (cm) & & 156.62 & 8.85 & & 148.48 & 7.71 & $0.111 \mathrm{a}$ \\
\hline \multirow{2}{*}{ Pre } & Edad (años) & & 13.26 & 0.45 & & 13.64 & 0.50 & $0.094 \mathrm{~b}$ \\
juvenil & Peso (kg) & \multirow{2}{*}{19} & 53.89 & 7.75 & \multirow{1}{*}{11} & 50.66 & 8.20 & $0.291 \mathrm{a}$ \\
& Talla (cm) & & 159.46 & 7.86 & & 159.23 & 7.91 & $0.938 \mathrm{a}$ \\
\hline \multirow{2}{*}{ Juvenil } & Edad (años) & & 15.72 & 1.07 & & 16.61 & 0.92 & $0.016 \mathrm{~b}^{*}$ \\
& Peso (kg) & \multirow{2}{*}{18} & 58.41 & 11.56 & \multirow{2}{*}{18} & 56.23 & 5.54 & $0.478 \mathrm{a}$ \\
& Talla (cm) & & 160.12 & 8.13 & & 161.67 & 5.21 & $0.501 \mathrm{a}$ \\
\hline
\end{tabular}

Tabla 2 .

Características antropométricas. *Diferencias significativas entre los GL1 y GL2 mediante la prueba de T-student (a) o la prueba U de Mann-Whitney (b).

\begin{tabular}{|c|c|c|c|c|c|c|}
\hline \multirow{2}{*}{ Categoría } & \multirow{2}{*}{$\begin{array}{l}\text { Nivel } \\
\text { Variable }\end{array}$} & \multicolumn{2}{|c|}{ GL1 } & \multicolumn{2}{|r|}{ GC2 } & \multirow[b]{2}{*}{ Valor $\mathrm{p}$} \\
\hline & & Media & $\begin{array}{c}\begin{array}{c}\text { Desviación } \\
\text { estándar }\end{array} \\
\end{array}$ & Media & $\begin{array}{c}\text { Desviación } \\
\text { estándar }\end{array}$ & \\
\hline \multirow{9}{*}{ Infantil } & Pantorrilla (cm) & 13.77 & 6.29 & 9.52 & 1.64 & $0.190 \mathrm{~b}$ \\
\hline & Muslo (cm) & 18.46 & 6.77 & 12.58 & 1.89 & $0.112 \mathrm{~b}$ \\
\hline & Porcentaje Graso (\%) & 23.44 & 6.56 & 17.66 & 2.02 & $0.083 \mathrm{~b}$ \\
\hline & Peso Graso $(\mathrm{kg})$ & 12.78 & 4.66 & 6.94 & 1.24 & $0.019 \mathrm{a}^{*}$ \\
\hline & Porcentaje Muscular (\%) & 39.84 & 5.91 & 41.82 & 1.35 & $0.362 \mathrm{a}$ \\
\hline & Peso Muscular (kg) & 20.88 & 3.35 & 16.44 & 2.24 & $0.022 \mathrm{a}^{*}$ \\
\hline & Porcentaje Oseo (\%) & 15.78 & 1.03 & 19.60 & 0.86 & $<0.001 a^{*}$ \\
\hline & Peso Oseo (kg) & 8.29 & 0.97 & 7.68 & 0.80 & $0.112 \mathrm{~b}$ \\
\hline & Corporal $\left(\mathrm{kg} / \mathrm{m}^{2}\right)$ & 21.48 & 2.26 & 17.74 & 1.03 & $0.005 \mathrm{a}^{*}$ \\
\hline \multirow{9}{*}{ Prejuvenil } & Pantorr & 12.73 & 6.58 & 11.70 & 4.40 & $0.832 \mathrm{~b}$ \\
\hline & & & & 15.22 & 4.79 & $0.237 \mathrm{a}$ \\
\hline & Porcen & 22.89 & 6.92 & 20.25 & 5.23 & $0.268 \mathrm{~b}$ \\
\hline & & 12.79 & 5.71 & 10.52 & 4.30 & $0.395 \mathrm{~b}$ \\
\hline & Porcentaje Muscular (\%) & 39.90 & 5.56 & 40.65 & 3.51 & $0.693 \mathrm{a}$ \\
\hline & Peso Mu & 21.23 & 2.54 & 20.43 & 2.82 & $0.428 \mathrm{a}$ \\
\hline & Porcen & 16.25 & 2.05 & 18.23 & 1.85 & $0.014 \mathrm{a}^{*}$ \\
\hline & Peso $\mathrm{O}$ & 8.64 & 0.88 & 9.13 & 0.96 & $0.166 \mathrm{a}$ \\
\hline & Corporal $\left(\mathrm{kg} / \mathrm{m}^{2}\right)$ & 21.28 & 3.49 & 19.99 & 3.08 & $0.319 \mathrm{a}$ \\
\hline \multirow{9}{*}{ Juvenil } & Pantorri & & 6.17 & 13.61 & 3.15 & $0.059 \mathrm{a}$ \\
\hline & Muslo ( & 22.35 & 6.63 & 17.86 & 3.64 & $0.009 \mathrm{~b}$ \\
\hline & Porcent & & 6.33 & 23.11 & 3.69 & $0.016 \mathrm{~b} * *$ \\
\hline & & 16.39 & 6.18 & 13.07 & 2.95 & $0.126 \mathrm{~b}$ \\
\hline & iscular (\%) & 35.95 & 6.77 & 38.79 & 2.81 & $0,181 \mathrm{~b}$ \\
\hline & Peso Muscular (kg) & 20.83 & 4.65 & 21.78 & 2.46 & 0.451 \\
\hline & Porcentaje Oseo (\%) & 15.44 & 1.96 & 17.21 & 1.80 & 0.008 \\
\hline & & 8.88 & 1.22 & 9.61 & 0.86 & $0.044 a^{*}$ \\
\hline & Índice de Masa Corporal $\left(\mathrm{kg} / \mathrm{m}^{2}\right)$ & 22.64 & 3.35 & 21.53 & 2.07 & 0.239 \\
\hline
\end{tabular}

Tabla 3

Características de las pruebas fisicas aplicadas. *Diferencias significativas entre los GL1 y GC2 mediante la prueba de T-student (a) o la prueba U de Mann-Whitney (b).

\begin{tabular}{|c|c|c|c|c|c|c|}
\hline \multirow{2}{*}{ Categoría } & \multirow{2}{*}{$\begin{array}{l}\text { Nivel } \\
\text { Variable }\end{array}$} & \multicolumn{3}{|c|}{ GL1 } & \multicolumn{2}{|l|}{$\mathrm{GC} 2$} \\
\hline & & Media & $\begin{array}{c}\text { Desviación } \\
\text { estándar }\end{array}$ & Media & $\begin{array}{l}\text { Desviación } \\
\text { estándar }\end{array}$ & Valor $\mathrm{p}$ \\
\hline \multirow{5}{*}{ Infantil } & Lanzamiento Balón Medicinal (m) & 3.31 & 0.64 & 3.48 & 0.61 & $0.630 \mathrm{a}$ \\
\hline & Salto en contra movimiento $(\mathrm{cm})$ & 19.08 & 3.58 & 25.58 & 3.70 & $0.007 \mathrm{a}^{*}$ \\
\hline & Abalakov & 22.16 & 5.98 & 22.92 & 3.76 & $0.802 \mathrm{a}$ \\
\hline & T test $\mathrm{p}$ & 12.57 & 1.07 & 12.06 & 0.30 & $0.208 \mathrm{a}$ \\
\hline & $T$ test $p$ & 2.48 & 0.89 & 11.77 & 0.58 & $0.141 \mathrm{a}$ \\
\hline \multirow{5}{*}{ Prejuvenil } & ledicinal (m) & 3.69 & 0.52 & 4.13 & 0.35 & $0.010 \mathrm{a}^{*}$ \\
\hline & Salto $\mathrm{e}$ & 20.92 & 4.45 & 28.27 & 6.17 & $0.001 \mathrm{a}^{*}$ \\
\hline & Abalakc & 25.04 & 4.96 & 25.68 & 4.99 & $0.737 \mathrm{a}$ \\
\hline & $\mathrm{T}$ test $\mathrm{p}$ & 12.06 & 1.00 & 11.36 & 0.88 & $0.065 \mathrm{a}$ \\
\hline & $\mathrm{T}$ test por & 12.13 & 0.68 & 11.47 & 0.99 & $0.070 \mathrm{a}$ \\
\hline \multirow{5}{*}{ Juvenil } & Lanzamien & & 0.50 & 4.17 & 0.60 & $0.094 \mathrm{a}$ \\
\hline & Salto en & 18.92 & 3.15 & 25.72 & 4.51 & $<0.01$ \\
\hline & Abalakov & 22.89 & 3.65 & 25.77 & 3.35 & $0.034 \mathrm{~b}^{*}$ \\
\hline & & 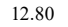 & 1.45 & 11.33 & 0.67 & 0.001 \\
\hline & $T$ test $\mathrm{pc}$ & 12.40 & 1.02 & 11.23 & 0.62 & \\
\hline
\end{tabular}

Tabla 4.

Valores de correlación ( $r$ ) entre variables físicas y antropométricas. *Correlaciones significativas a un nivel de 0.05 . **Correlaciones significativas a un nivel de 0.01 .

\begin{tabular}{lcccc} 
& Pantorrilla & Muslo & $\begin{array}{c}\text { Porcentaje } \\
\text { Graso }\end{array}$ & $\begin{array}{c}\text { Porcentaje } \\
\text { Muscular }\end{array}$ \\
\hline Salto en contra movimiento, CMJ & $-0.477^{* *}$ & $-0.496^{* *}$ & $-0.550^{* *}$ & $0.452^{* *}$ \\
Abalakov & $-0.443^{* *}$ & $-0.356^{*}$ & $-0.394^{*}$ & 0.290 \\
T test por derecha & $0.350^{* *}$ & $0.457^{* *}$ & $0.428^{*}$ & $-0.378^{*}$ \\
T test por izquierda & $0.604^{* *}$ & $0.604^{* *}$ & $0.645^{* *}$ & $-0.615^{* *}$
\end{tabular}

tes, sino también en la capacidad de vencer al contrario lo cual genera un contexto de juego variante, en donde cada jugador tomará decisiones y acciones utilizando las capacidades coordinativas y condicionales (Alarcón López et al., 2010).

En el presente estudio uno de los principales hallazgos fue la diferencia significativa $(p<0,01)$ en los niveles de fuerza explosiva en el tren inferior, evaluada por medio de la prueba de salto en contra movimiento, $\mathrm{CMJ}$, en las tres categorías, entre el GL1 y el GC2 (ver tabla 3). De este resultado se puede interpretar que la mayor intensidad horaria semanal de entrenamiento afecta el desempeño en esta prueba. Es decir, el mayor número de horas de entrenamiento semanal 
de las jugadoras del GL1 se relaciona con una menor altura lograda en el CMJ, comparativamente que las jugadoras del GC2, en las tres categorías.

Las características antropométricas de la masa corporal y la talla son importantes en el baloncesto, el peso influye en el rendimiento físico, el ser alto puede facilitar la tarea de recuperar el balón (Gilabert Labra et al., 2014). Al comparar los resultados de la masa corporal y la altura de este estudio con el crecimiento de los estándares regulatorios de las niñas colombianas (Briceño et al., 2012), las jugadoras de baloncesto jóvenes de cada uno de los niveles, excepto la categoría infantil, tenía una masa corporal y un tamaño superior al percentil 50 de las niñas colombianas, porque según las normas reportadas por Briceño et al. (2012), las niñas de 12 deben tener un peso de $44 \mathrm{~kg}$ y un tamaño de $151 \mathrm{~cm}$, a los 13 años $48 \mathrm{~kg}$ y $155 \mathrm{~cm}$ durante 16 años y $52 \mathrm{~kg}$ y $158 \mathrm{~cm}$, sin embargo, es necesario aclarar que estos estándares se aplican a las niñas sanas, pero no son atletas. Además, los hallazgos de esta investigación son similares a los encontrados en otro estudio (Pereira Gaspar, 2002) que evaluó a jugadoras de baloncesto portugueses de 15 a 16 años y encontró una masa corporal promedio de 54,91 $\pm 8,24 \mathrm{~kg}$ y un tamaño de $160,59 \pm 5,26 \mathrm{~cm}$. Estos hallazgos y los del presente estudio son más bajos que los obtenidos por una investigación en jóvenes chilenas de baloncesto (14,5 $\pm 0,92$ años) (Labra et al., 2014) quienes tuvieron un peso promedio de $58,1 \mathrm{~kg}$ $( \pm 8,41 \mathrm{~kg})$.

Los hallazgos de la composición corporal tanto para el GL1 como el GC2 difieren de los resultados encontrados en un estudio con jugadoras españolas (Sánchez et al., 2009), donde los porcentajes de grasa fueron menores que el grupo evaluado $12,0 \%$ ( $\pm 2,1 \%$ ) (B Women's League), $13,8 \%$ ( $\pm 2,4 \%$ ) (League Femina 2) y $12,4 \% \pm 2,0 \%$ (First National) y los porcentajes de masa muscular fueron 43,3\% ( $\pm 3,3 \%)$ (LFB ), $44,4 \%( \pm 3,8 \%)(\mathrm{LF} 2)$ y $45,8 \%( \pm 3,4 \%)(\mathrm{PN})$. Sin embargo, los porcentajes de grasa en las categorías pre-juvenil y juvenil, en ambos grupos de los jugadores de baloncesto de Bogotá fueron más bajos que los resultados encontrados por Labra y colaboradores (Labra et al., 2014), quienes encontraron porcentajes de 30.8 para jugadores grasos chilenos entre 13 y 15 años. Además, la comparación de los hallazgos en la composición corporal del grupo de la liga presentó dos niveles de registros más altos, mientras que el grupo escolar, informó valores más bajos, con diferencias significativas entre las diversas categorías para los componentes de la masa grasa y la masa muscular, lo que puede ayudar a su rendimiento físico, porque según Silva et al. (Silva et al., 2012), para aumentar la masa corporal magra y reducir la masa grasa en los jóvenes jugadores de baloncesto de Portugal, la fuerza y la potencia aumentan un 14,4 y un $9,8 \%$ respectivamente.

En cuanto la influencia de las variables antropométricas en el rendimiento del baloncesto en jugadores profesionales de elite Baloncesto, un estudio se llevó a cabo aplicando dos evaluaciones prestest coincidiendo con la pretemporada y postest después de 14 días de entrenamiento (Albaladejo et al., 2019). Los resultados más relevantes se presentaron en una disminución del peso, en el pliegue abdominal y muslo, porcentaje y peso graso, como también un incremento en el porcentaje de masa muscular.
En relación con las variables de condición física el GC2 presento mejores resultados de fuerza explosiva, debido posiblemente al menor estrés relacionado con el volumen de entrenamiento. En el ámbito internacional, específicamente para la fuerza explosiva en el baloncesto femenino, lo primero que se nota es que la mayoría de los estudios se han desarrollado en hombres, sin embargo, un estudio llevado a cabo con 16 jugadores de baloncesto de la selección chilena (García Guajardo et al., 2010), cuya edad promedio fue de 16,9 $\pm 1,3$ años, reportaron para el salto en contra movimiento $(29,0 \mathrm{~cm} \pm 5,1 \mathrm{~cm})$ y salto de abalakov $(31,1 \mathrm{~cm} \pm 5,3 \mathrm{~cm})$ datos superiores a los registrados en los dos niveles en las jugadoras de Bogotá (CL1: CMJ 19,3; 22,6 salto de abalakov, GC2: salto de abalakov y CMJ 25,7;25,8), mientras que en jugadores de baloncesto europeas (Erculj et al., 2010) los resultados de las pruebas de salto en contra movimiento se encontró valores entre $26.34 \mathrm{a} 27.59 \mathrm{~cm}$, similares a los realizados por el GC2 (25,6 a $28,3 \mathrm{~cm})$.

Además de lo anterior, las categorías infantil y prejuvenil del presente estudio obtuvieron mejores resultados en la prueba de salto en contra movimiento con respecto a la categoría juvenil, sin embargo, se espera que los juveniles tenga los mejores resultados, según lo mencionado por una revisión de estudios observacionales y experimentales (Ziv \& Lidor, 2010) dónde los factores de desarrollo como los cambios en la maduración de los jugadores influyen en el aumento de la ejecución del salto vertical. Lo anterior se ha comprobado en jóvenes mayores de 18 años, jugadores de baloncesto de Túnez (Abdelkrim et al., 2010), dónde se evidenció una mejora progresiva en varias pruebas de agilidad, fuerza explosiva y velocidad $(\mathrm{p}<0,05)$, con respecto a los menores de 18 años quienes tenían un menor volumen, intensidad y carga de preparación en el deporte. Estos últimos factores pueden estar involucrados en el resultado de los jóvenes jugadores de baloncesto estudiados, posiblemente el proceso de entrenamiento o el mismo estilo de vida en esta edad hace que el rendimiento no sea mejor que el pre-juvenil, por lo tanto, se recomienda para futuras investigaciones tener en cuenta estos dos aspectos.

La fuerza explosiva del tren superior tuvo resultados superiores en el GC2, en comparación con los de GL1, sin embargo difieren de los hallados con jugadoras de baloncesto europeo de 14 años (Erculj et al., 2010) en la prueba de lanzamiento de balón medicinal con registros entre 4,27 a 4,66 m, mayores a los obtenidos por las jugadores del presente estudio de la misma categoría y en ambos grupos.

En la prueba de agilidad, los mejores registros fueron para el GC2, presentando menores tiempos de ejecución a nivel juvenil (11,3 $\mathrm{s} \pm 0,7 \mathrm{~s})$. Los tiempos no difieren mucho de los encontrados por (Zariæ, 2014) que realizaron una prueba previa y posterior a un entrenamiento de seis semanas con jugadoras de 17 años de Serbia. Estos mejoraron su rendimiento al pasar de $11,477 \mathrm{~s}( \pm 0,784 \mathrm{~s})$ a $10,731 \mathrm{~s}( \pm 0,625 \mathrm{~s})$. También un estudio con jugadoras Sub 16 de España (FortVanmeerhaeghe et al., 2016), mostraron un rendimiento en el $\mathrm{T}$ test mejor a las reportadas en el presente estudio con una media de 11,04 s ( $\pm 0,66 \mathrm{~s})$. Estos resultados, sugieren que el rendimiento físico del GC2 se acerca más a los encontrados por jóvenes basquetbolistas de contextos internacionales en comparación con los resultados obtenidos por el GL1. 
Dentro de las limitaciones del presente estudio se encuentran un tamaño de muestra reducido, especialmente en la categoría infantil, aunque en las demás categorías tampoco es posible generalizar los hallazgos y la poca evidencia encontrada de estudios afines con muestras similares. Este último argumento también puede verse como una oportunidad para que los resultados del presente estudio sean discutidos en futuras investigaciones con diseños prospectivos.

\section{Conclusiones}

Esta investigación encontró mejores resultados en el GC2 en las variables de fuerza explosiva, agilidad, menores porcentajes grasos y mayores pesos óseos, especialmente en la categoría juvenil con respecto al GL1. Sin embargo, el GC2 entrenan dos horas menos que GC1, ahora otra posible explicación puede deberse a las mejores condiciones socio económicas que posiblemente presentan las jóvenes basquetbolistas pertenecientes al GC2, ya que es característico que la asociación de colegios internacionales de Bogotá, Uncoli, sea integrada por instituciones educativas cuyos estudiantes son provenientes de familias de medianos a altos ingresos económicos, lo que no necesariamente puede ocurrir con las jugadoras pertenecientes al GL1.

\section{Referencias}

Abdelkrim, N. B., Chaouachi, A., Chamari, K., Chtara, M., \& Castagna, C. (2010). Positional role and competitive-level differences in elite-level men's basketball players. The Journal of Strength \& Conditioning Research, 24(5), 1346-1355.

Alarcón López, F., Cárdenas Vélez, D., Miranda León, M. T., Ureña Ortín, N., \& Piñar López, M. I. (2010). La influencia de un modelo constructivista para la enseñanza de la táctica en baloncesto sobre la eficacia del juego durante la competición. Retos. Nuevas Tendencias En Educación Física, Deporte y Recreación, 17, 15-20.

Albaladejo, M., Vaquero-Cristóbal, R., \& Esparza-Ros, F. (2019). Efecto del entrenamiento en pretemporada en las variables antropométricas y derivadas en jugadores de baloncesto de élite (Effect of preseason training on anthropometric and derived variables in professional basketball players) Retos, 36(36), 474-479.

Baechle, T. R., \& Earle, R. W. (2008). Essentials of strength training and conditioning. Human kinetics.

Blanco, R. J., \& Maya, M. J. (2008). Fundamentos de Salud Pública. Administración de Servicios de Salud.: Vol. II. Corporación para Investigaciones Biológicas.

Bosco, C. (2000). La fuerza muscular: Aspectos metodológicos (Vol. 307), Inde.

Bosco, C., \& Riu, J. M. P. (1994). La valoración de la fuerza con el test de Bosco. Paidotribo Barcelona.

Briceño, G., Durán, P., Colón, E., Line, D., Merker, A., Abad, V., Chahín, S. Del Toro, K., Matallana, A., \& Llano, M. (2012). Protocolo del estudio para establecer estándares normativos de crecimiento de niños colombianos sanos. Pediatría, 45(4), 235-242.

Erculj, F., Blas, M., \& Bracic, M. (2010). Physical Demands on Young Elite European Female Basketball Players With Special Reference to Speed, Agility, Explosive Strength, and Take-off Power. The Journal of Strength \& Conditioning Research, 24(11), 2970. https://doi.org/10.1519/ JSC.0b013e3181e38107

Fort-Vanmeerhaeghe, A., Montalvo, A., Latinjak, A., \& Unnithan, V. (2016) Physical characteristics of elite adolescent female basketball players and their relationship to match performance. Journal of Human Kinetics, 53(1), 167-178. https://doi.org/10.1515/hukin-2016-0020

García Guajardo, V., De Arruda, M., Aránguiz Aburto, H., Rojas Díaz, S., \& García Krauss, P. (2010). Características antropométricas, composición corporal, somatotipo y rendimiento anaeróbico y aeróbico de mujeres juveniles baloncestistas chilenas. Educación Física y Deporte, 29(2), 255-265.

Garcia-Gil, M., Torres-Unda, J., Esain, I., Duñabeitia, I., Gil, S. M., Gil, J., \& Irazusta, J. (2018). Anthropometric Parameters, Age, and Agility as Performance Predictors in Elite Female Basketball Players. Journal of
Strength and Conditioning Research, 32(6), 1723-1730. https://doi.org/ 10.1519/JSC.0000000000002043

Gilabert Labra, Á. A., Retamales Muñoz, F. J., Castillo Retamal, M., \& Valenzuela Bustamante, R. A. (2014). Perfil somatotípico y composición corporal de basquetbolistas escolares chilenas de 13 a 15 años medallistas en fase regional a los juegos escolares 2011. Revista Ciencias de La Actividad Física UCM, 1, 15

González Espinosa, S., Ibáñez Godoy, S. J., Feu Molina, S., \& Galatti, L. R. (2017). Programas de intervención para la enseñanza deportiva en el contexto escolar, PETB y PEAB: Estudio preliminar.

Labra, Á. A. G., Muñoz, F. J. R., Retamal, M. C., \& Bustamante, R. A. V. (2014). Perfil somatotípico y composición corporal de basquetbolistas escolares chilenas de 13 a 15 años medallistas en fase regional a los Juegos Escolares 2011. Revista Ciencias de La Actividad Física, 15(1), 33-42.

Little, T., \& Williams, A. G. (2005). Specificity of acceleration, maximum speed, and agility in professional soccer players. Journal of Strength and Conditioning Research, 19(1), 76-78. https://doi.org/10.1519/14253.1

Martinez-López, E. J. (2002). Pruebas de aptitud física. Editorial Paidotribo.

Masanovic, B. (2018). Comparative study of anthropometric measurement and body composition between junior basketball and volleyball players from Serbian national league. Sport Mont, 16(3), 19-24.

Narazaki, K., Berg, K., Stergiou, N., \& Chen, B. (2009). Physiological demands of competitive basketball. Scandinavian Journal of Medicine \& Science in Sports, 19(3), 425-432. https://doi.org/10.1111/j.16000838.2008.00789.x

Ostojic, S. M., Mazic, S., \& Dikic, N. (2006). Profiling in basketball: Physical and physiological characteristics of elite players. Journal of Strength and Conditioning Research, 20(4), 740.

Otero, F. M., Carmona, J., Albornoz, M., Calvo, A., \& Díaz, J. A. (2014). Metodología de enseñanza de los deportes de invasión en primaria/Teacher's methodology of invasion games in primary school. Revista Internacional de Medicina y Ciencias de La Actividad Fisica y Del Deporte, 53.

Pauole, K., Madole, K., Garhammer, J., Lacourse, M., \& Rozenek, R. (2000). Reliability and validity of the T-test as a measure of agility, leg power, and leg speed in college-aged men and women. The Journal of Strength \& Conditioning Research, 14(4), 443-450.

Peña López, J., Moreno-Doutres, D., Coma Bau, J., \& Cook, M. (2018). Anthropometric and fitness profile of high-level basketball, handball and volleyball players.

Pereira Gaspar, P. (2002). Evaluación corporal en atletas jóvenes de baloncesto femenino. Revista Internacional de Medicina y Ciencias de La Actividad Física y El Deporte, 2. http://cdeporte.rediris.es/revista/revista6/ evaluacion.htm

Pizzigalli, L., Micheletti Cremasco, M., LA Torre, A., Rainoldi, A., \& Benis, R. (2017). Hand grip strength and anthropometric characteristics in Italian female national basketball teams. The Journal of Sports Medicine and Physical Fitness, 57(5), 521-528. https://doi.org/10.23736/S00224707.16.06272-1

Pojskic, H., Sisic, N., Separovic, V., \& Sekulic, D. (2018). Association Between Conditioning Capacities and Shooting Performance in Professional Basketball Players: An Analysis of Stationary and Dynamic Shooting Skills. Journal of Strength and Conditioning Research, 32(7), 19811992. https://doi.org/10.1519/JSC.0000000000002100

Sánchez, I. S., Campo, S. S., de Benito Trigueros, A., Velasco, J. M. I., \& Sáenz, G. C. (2009). Perfil antropométrico de las jugadoras de baloncesto españolas: Análisis en función del nivel competitivo y de la posición específica de juego. RICYDE. Revista Internacional de Ciencias Del Deporte, 5(15), 1-16.

Silva, A. M., Santos, D. A., Matias, C. N., Rocha, P. M., Petroski, E. L., Minderico, C. S., \& Sardinha, L. B. (2012). Changes in regional body composition explain increases in energy expenditure in elite junior basketball players over the season. European Journal of Applied Physiology, 112(7), 2727-2737. https://doi.org/10.1007/s00421-011-2221-8

Vaquera, A., Santos, S., Villa, J. G., Morante, J. C., \& García-Tormo, V. (2015). Anthropometric Characteristics of Spanish Professional Basketball Players. Journal of Human Kinetics, 46, 99-106. https://doi.org/10.1515/ hukin-2015-0038

Young, W. B., Dawson, B., \& Henry, G. J. (2015). Agility and change-ofdirection speed are independent skills: Implications for training for agility in invasion sports. International Journal of Sports Science \& Coaching, 10(1), 159-169.

Zariæ, I. (2014). The effects of a six-week training program on motor and functional skills of female basketball players. Fizièka Kultura, 68(1), 7582.

Ziv, G., \& Lidor, R. (2010). Vertical jump in female and male basketball players - A review of observational and experimental studies. Journal of Science and Medicine in Sport, 13(3), 332-339. 